\title{
A QUASILINEAR DELAYED HYPERBOLIC NAVIER-STOKES SYSTEM: GLOBAL SOLUTION, ASYMPTOTICS AND RELAXATION LIMIT*
}

\author{
ALEXANDER SCHÖWE†
}

\begin{abstract}
We consider a hyperbolic quasilinear fluid model, that arises from a delayed version for the constitutive law for the deformation tensor in the incompressible Navier-Stokes equation. We prove global existence of small solutions and asymptotic results in $\mathbb{R}^{3}$ and the half-space with slip boundary conditions. Futhermore we show that this relaxed system is close to the classical Navier-Stokes equation in the sense that for small times $t$ the solutions converge in high Sobolev norms to the solution of the incompressible Navier-Stokes equation.
\end{abstract}

Key words. Navier-Stokes, global small solution, decay rate, Cattaneo law, Fourier law, Oldroyd, relaxation limit, delay equation.

AMS subject classifications. 35L72, 35Q30, 35Q35, 76D05, 35B40.

1. Introduction. Let $n \geq 2$ and $T, \tau, \mu>0$. In this note the fluid model

$$
\begin{aligned}
\tau u_{t t}-\mu \Delta u & +u_{t}+\nabla p+\tau \nabla p_{t} & & \\
& =-(u \cdot \nabla) u-\left(\tau u_{t} \cdot \nabla\right) u-(\tau u \cdot \nabla) u_{t} & & \text { in }(0, T) \times \mathbb{R}^{n}, \\
\operatorname{div} u & =0 & & \text { in }(0, T) \times \mathbb{R}^{n}, \\
u(0, \cdot) & =u_{0}, \quad u_{t}(0, \cdot)=u_{1} & & \text { in } \mathbb{R}^{n}
\end{aligned}
$$

for the velocity field $u=u(t, x):(0, T) \times \mathbb{R}^{n} \rightarrow \mathbb{R}^{n}$ and the pressure $p=p(t, x)$ : $(0, T) \times \mathbb{R}^{n} \rightarrow \mathbb{R}$, where $u_{0}$ and $u_{1}$ are given initial data, will be considered. This model arises from a delayed version for the constitutive law for the deformation tensor in the incompressible Navier-Stokes equation (compare below).

First of all there will be given some well-posedness and asymptotic results to the model above in $\mathbb{R}^{3}$, that improve and complete known results due to Racke and Saal from [11] and [12].

Furthermore, thanks to a reflection technique (cp. [15]), the equation in the half-space $\mathbb{R}_{+}^{n}$ with slip-boundary conditions can also be solved.

As another main result it will be shown, that the model above is closely related to the incompressible Navier-Stokes equation, in the sense that for small times $t$ the solutions $\left(u^{\tau}\right)_{\tau}$ of $(1)$ corresponding to $\tau>0$ converge for $\tau \rightarrow 0$ in high Sobolev norms to the solution of the incompressible Navier-Stokes equation. follows.

The model can be derived from the incompressible Navier-Stokes equation as

The incompressible Navier-Stokes equation (in the following also referred to as classical Navier-Stokes equation) is given by

$$
\begin{aligned}
u_{t}+(u \cdot \nabla) u+\nabla p & =\operatorname{div} 2 S & & \text { in }(0, T) \times \Omega, \\
\operatorname{div} u & =0 & & \text { in }(0, T) \times \Omega, \\
\left.u\right|_{t=0} & =u_{0} & & \text { in } \Omega,
\end{aligned}
$$

\footnotetext{
*Received February 15, 2012; accepted for publication September 4, 2012.

$\dagger$ University of Konstanz, Department of Mathematics and Statistics, 78464 Konstanz, Germany (Alexander.Schoewe@uni-konstanz.de).
} 
where the deformation tensor $S$ is determined by

$$
S=\frac{\mu}{2}\left(\nabla u+(\nabla u)^{\prime}\right) .
$$

Like the change from Fourier type law to Cattaneo in heat conduction (cp. [4]), this law is replaced by a delayed version

$$
S+\tau S_{t}=\frac{\mu}{2}\left(\nabla u+(\nabla u)^{\prime}\right),
$$

for a small relaxation parameter $\tau>0$, where the left hand side is a formal first order Taylor approximation of $S(t+\tau)$. By the second line in (2) one gets

$$
\operatorname{div} 2\left(S+\tau S_{t}\right)=\mu \Delta u
$$

and therefore (1) can be derived by differentiating the first line in (2) with respect to $t$ and after a multiplication with $\tau$ adding the resulting line to the original one. The equation (1) will be refered to as hyperbolic Navier-Stokes equation.

This model was already derived in [2] and [3]. But in a mathematical rigorous way, concerning well-posedness in high Sobolev norms, it has been treated first in [11] an [12]. Further results have been established in the diploma thesis of the author [13], which in parts will be presented in the following.

Paicu and Raugel considered in [9] another hyperbolic fluid model by just adding a hyperbolic perturbation $\tau u_{t t}$ to the classical Navier-Stokes equation, but this model rather differs to the one above, because their model remains semilinear, which makes it possible to carry over the proofs for the classical Navier-Stokes equation. For an introduction to the Navier-Stokes equation we refer to [14] and [7].

Furthermore in [1] the model from [9] was examined for $\tau \rightarrow 0$ and it was shown, that the solutions $\left(u^{\tau}\right)_{\tau}$ corresponding to $\tau>0$ converge for $\tau \rightarrow 0$ to the solution of the incompressible Navier-Stokes equation. However this result on an approximation of the classical Navier-Stokes equation by relaxation cannot be compared to the Theorem 3.1 in this note, because different systems on different domains are considered. Formally, regarding the derivation of the hyperbolic Navier-Stokes equation, the result on the approximation seems to be not so astonishing but one has to keep in mind the works [5] and [6], where it was shown that delayed systems, that are formally close together, can behave differently. For example in [6] it was shown, that a equation, coming from a Cattaneo type law, might not be exponentially stable, although the same system with a Fourier type law is. In [5] it is even shown, that formal high taylor expansions of the delayed term, can lead to ill-posedness. Altogether the result on the approximation feeds hope to gain new ideas for the classical Navier-Stokes equation by examination of the hyperbolic Navier-Stokes equation. Futhermore it makes the conjecture of Racke and Saal in [12], that the hyperbolic Navier-Stokes equation has a blow-up for large data, even more interesting.

The paper is organized as follows. In section two well-posedness and asymptotic results to the model (1) will be proven, where the whole space is treated in subsection 2.1 and the half-space in subsection 2.2 .

In section three we will prove the result on the convergence to the classical NavierStokes equation for $\tau \rightarrow 0$. In the first part of the section the boundedness in high Sobolev norms of $\left(u^{\tau}\right)_{\tau}$ for small times $t$ in $\tau$ is proven, which makes it possible to show in the second part the convergence to the solution of the classical Navier-Stokes equation. 
2. Well-posedness and asymptotic results. In this section there will be given some well-posedness and asymptotic results for (1), but first of all some remarks on the notation used in this note.

Let $X$ be a general Banach space and $\Omega \subseteq \mathbb{R}^{n}$ a set, then $C^{m}(\Omega, X)$ denotes the space of $\mathrm{m}$-times continuously differentiable functions with values in $X$. Analogously $L^{p}(\Omega, X)$ with $1 \leq p \leq \infty$ denotes the standard Lebesgue space of $\mathrm{X}$-valued functions with norm $\|\cdot\|_{p}$. For the Hilbert space $L^{2}(\Omega, X)$ we write $\langle\cdot, \cdot\rangle$ for the scalar product.

As usual $W^{k, p}(\Omega, X)$ denotes the Sobolev space of $\mathrm{k}$-th order, with the norm

$$
\|u\|_{W^{m, p}}:=\|u\|_{m, p}:= \begin{cases}\left(\sum_{0 \leq|\alpha| \leq m}\left\|\partial^{\alpha} u\right\|_{p}^{p}\right)^{\frac{1}{p}}, & \text { for } \leq p<\infty \\ \max _{0 \leq|\alpha| \leq m}\left\|\partial^{\alpha} u\right\|_{\infty}, & \text { for } p=\infty\end{cases}
$$

where the multi-index notation is used, that means for $\alpha \in \mathbb{N}_{0}^{n}$ we set $\nabla^{\alpha}:=\partial^{\alpha}:=$ $\partial_{1}^{\alpha_{1}} \cdot \ldots \cdot \partial_{n}^{\alpha_{n}}$.

For a vector-valued function $u: \Omega \subseteq \mathbb{R}^{n} \rightarrow \mathbb{R}^{n}, x \mapsto u(x)=\left(u_{1}(x), \ldots, u_{n}(x)\right) u_{i}$ denotes the $i$-th component.

The Helmholtz projection onto the space

$$
L_{\sigma}^{q}(\Omega):={\overline{\left\{u \in\left(C_{0}^{\infty}(\Omega)\right)^{n}: \operatorname{div} u=0\right\}}}^{\|\cdot\|_{q}} \quad \text { for } \Omega \in\left\{\mathbb{R}^{n}, \mathbb{R}_{+}^{n}\right\}
$$

is denoted by $P$.

2.1. Whole space. In [11] the well-posedness in the whole space $\mathbb{R}^{n}$ has been proven under a smallness condition on the initial data, where the existence time depends on the highest norm of the initial data. One can improve this dependence to the result

Theorem 2.1 (Local existence). Let $n \geq 2$ and $s \geq m>\frac{n}{2}$. For all

$$
\left(u_{0}, u_{1}\right) \in\left(W^{s+2,2}\left(\mathbb{R}^{n}\right) \cap L_{\sigma}^{2}\left(\mathbb{R}^{n}\right)\right) \times\left(W^{s+1,2}\left(\mathbb{R}^{n}\right) \cap L_{\sigma}^{2}\left(\mathbb{R}^{n}\right)\right)
$$

there exists a $T>0$ and a unique solution $(u, p)$ to (1) satisfying

$$
\begin{gathered}
u \in C^{0}\left([0, T], W^{s+2,2}\left(\mathbb{R}^{n}\right) \cap L_{\sigma}^{2}\left(\mathbb{R}^{n}\right)\right) \cap C^{1}\left([0, T], W^{s+1,2}\left(\mathbb{R}^{n}\right)\right) \cap C^{2}\left([0, T], W^{s, 2}\left(\mathbb{R}^{n}\right)\right), \\
\nabla\left(p+\tau p_{t}\right) \in C^{0}\left([0, T], W^{s, 2}\left(\mathbb{R}^{n}\right)\right) .
\end{gathered}
$$

The existence time $T$ only depends on $\left\|u_{0}\right\|_{m+2,2}$ and $\left\|u_{1}\right\|_{m+1,2}$.

Hints on the proof. One has to check where in [11] the existence time is determined. The basic idea of the proof in [11] is to construct solutions to a linearized system and then use Majda's fixed point iteration to treat the full system. To this end the boundedness of the iteration sequence is needed, which can only been proven with a restriction to the existence time (compare [11, Lemma 5.2]). Therefore one has to find a better proof of [11, Lemma 5.2].

One approximates the initial data with smooth functions, then gets a smoother iteration sequence, which justifies to carry over the first part of the calculations in $[11$, Theorem 4.5 Step 2], and finally gets with the idea used in [10, Theorem 5.8] the desired energy estimate with better dependence of the existence time. The convergence of the iteration sequence to the solution can be proven like in [11].

In [12] the global existence in $\mathbb{R}^{2}$ and $\mathbb{R}^{3}$ was proven with a method by Klainerman and Ponce, like it is for example described in [10]. The proof uses convergence rates 
coming from the damped wave equation, which in two dimensions are worse than in three dimensions and therefore Racke and Saal focused on the two dimensional case. Actually if one compares the equation with the damped wave equation, where quadratic nonlinearties in $\mathbb{R}^{2}$ touch the critical borderline, one would expect that the two dimensional case is more complicated (compare $[12,1$. Introduction]). The remark in [12] that the result in $\mathbb{R}^{3}$ can be improved has now been carried out in detail and the following Theorem was proven.

THEOREM 2.2 (Global solution). Let $m \geq 3$, then there exists a $\delta>0$ such that for initial data $\left(u_{0}, u_{1}\right) \in\left(W^{m+3,2}\left(\mathbb{R}^{3}\right) \cap L_{\sigma}^{2}\left(\mathbb{R}^{3}\right)\right) \times\left(W^{m+2,2}\left(\mathbb{R}^{3}\right) \cap L_{\sigma}^{2}\left(\mathbb{R}^{3}\right)\right)$ satisfying

$$
\left\|u_{0}\right\|_{m+3,2}+\left\|u_{1}\right\|_{m+2,2}+\left\|u_{0}\right\|_{1}+\left\|u_{1}\right\|_{1}<\delta
$$

there exists a unique global solution $(u, p)$ to the hyperbolic Navier-Stokes equation (1) with

$$
\begin{array}{r}
u \in C^{0}\left([0, \infty), W^{m+3,2}\left(\mathbb{R}^{3}\right) \cap L_{\sigma}^{2}\left(\mathbb{R}^{3}\right)\right) \\
\cap C^{1}\left([0, \infty), W^{m+2,2}\left(\mathbb{R}^{3}\right)\right) \\
\cap C^{2}\left([0, \infty), W^{m+1,2}\left(\mathbb{R}^{3}\right)\right),
\end{array}
$$

$$
\nabla\left(p+\tau p_{t}\right) \in C^{0}\left([0, \infty), W^{m+1,2}\left(\mathbb{R}^{3}\right)\right)
$$

Furthermore

$$
\begin{aligned}
\|u(t)\|_{m, 2}=\mathcal{O}\left(t^{-\frac{3}{4}}\right), & \left\|u_{t}(t)\right\|_{m, 2}=\mathcal{O}\left(t^{-\frac{3}{2}}\right), \\
\|\nabla u(t)\|_{m, 2}=\mathcal{O}\left(t^{-\frac{5}{4}}\right) & \text { for } t \rightarrow \infty
\end{aligned}
$$

holds.

REMARK 2.3 (Improvements). Obviously the decay rates are better than in the two dimensional case, but furthermore the conditions on the initial conditions were weakened and the proof was shortened and therefore the Theorem is a interesting result. Of course it is also possible to show $\|\cdot\|_{m_{1}, q^{-}}$decay rates like in [12], but for the proof of the global existence in $\mathbb{R}^{3}$ this is not necessary.

Proof of Theorem 2.2. One applies the Helmholtz projection $P$ to (1) and gets for $u \in L_{\sigma}^{2}\left(\mathbb{R}^{3}\right)$ the equation

$$
\begin{array}{rlrl}
\tau u_{t t}-\mu \Delta u+u_{t}= & -P((u \cdot \nabla) u)-P\left(\left(\tau u_{t} \cdot \nabla\right) u\right) & \\
& -P\left((\tau u \cdot \nabla) u_{t}\right) \equiv N_{1}+N_{2}+N_{3} & & \text { in }(0, \infty) \times \mathbb{R}^{3}, \\
u(0, \cdot)=u_{0}, \quad u_{t}(0, \cdot)=u_{1} & \text { in } \mathbb{R}^{3} .
\end{array}
$$

According to Theorem 2.1 let $u$ be the local solution to (11). Then as usual the proof consists of a high energy estimate and a weighted a priori estimate for $u$, which together guarantee that the local existence theorem can be applied successively to obtain a global solution.

The high energy estimate can be quoted from [12, Theorem 4.1].

Theorem 2.4 (High energy estimate). For

$$
E_{m}(t):=E_{m}(u(t)):=\frac{1}{2} \sum_{|\alpha| \leq m+1}\left(\tau\left\|\nabla^{\alpha} u_{t}\right\|_{2}^{2}+\mu\left\|\nabla^{\alpha} \nabla u\right\|_{2}^{2}+\varepsilon_{2}\left\|\nabla^{\alpha} u\right\|_{2}^{2}\right)(t)
$$


with $\varepsilon_{2}>0$ appropriate, there exists a $c>0$ independent from $T$ and the initial data $\left(u_{0}, u_{1}\right) \in\left(W^{m+2,2} \cap L_{\sigma}^{2}\right) \times\left(W^{m+1,2} \cap L_{\sigma}^{2}\right)$ such that for $0 \leq t \leq T$ and $m>\frac{3}{2}+1$

$$
E_{m}(t) \leq c E_{m}(0) e^{c \int_{0}^{t}\left(\|u\|_{\infty}^{2}+\left\|u_{t}\right\|_{1, \infty}+\|\nabla u\|_{\infty}\right)(r) \mathrm{d} r}
$$

holds.

The improvement in the proof of the global existence in the three dimensional case to [12] lies in the weighted a priori estimate, that thanks to better decay rates, can be formulated and proven easier (compare the following Theorem to $[12$, Theorem $5.3])$.

THEOREM 2.5 (Weighted a priori estimate). Let $m>\frac{3}{2}+1$, then there exists a $\delta_{1}>0$ such that for initial data $\left(u_{0}, u_{1}\right) \in\left(W^{m+3,2} \cap L_{\sigma}^{2}\right) \times\left(W^{m+2,2} \cap L_{\sigma}^{2}\right)$ with

$$
\left\|u_{0}\right\|_{m+3,2}+\left\|u_{1}\right\|_{m+2,2}+\left\|u_{0}\right\|_{1}+\left\|u_{1}\right\|_{1}<\delta_{1},
$$

there exists a $M_{0}>0$ independent from $T$, such that for the solution $u$ of (1)

$$
\begin{aligned}
M(T): & =\sup _{0 \leq t \leq T}\left\{(1+t)^{\frac{3}{4}}\|u(t)\|_{m, 2}+(1+t)^{\frac{3}{2}}\left\|u_{t}(t)\right\|_{m, 2}+(1+t)^{\frac{5}{4}}\|\nabla u(t)\|_{m, 2}\right\} \\
& \leq M_{0}
\end{aligned}
$$

holds.

Proof. We quote the representation formula [12, Lemma 5.2] for the solution $u$

Lemma 2.6 (Representation formula). Let $w(t) g$ denote the solution to

$$
\begin{array}{cc}
\tau v_{t t}-\mu \Delta v+v_{t}=0 & \text { in }(0, \infty) \times \mathbb{R}^{3}, \\
v(0, \cdot)=0, \quad v_{t}(0, \cdot)=g & \text { in } \mathbb{R}^{3} .
\end{array}
$$

Then $u$ can be represented as

$$
u(t)=w(t)\left(u_{1}+\frac{1}{\tau} u_{0}\right)+\partial_{t} w(t) u_{0}+\frac{1}{\tau} \int_{0}^{t} w(t-r) \sum_{j=1}^{3} N_{j}(r) \mathrm{d} r .
$$

which makes it possible two obtain convergence rates for the solution $u$ from the damped wave equation. In the case of $\mathbb{R}^{3}$ one only (compare to [12, Lemma 5.1]) needs the following convergence rates.

LEMMA 2.7. Let $v$ denote the solution to

$$
\begin{array}{cc}
\tau v_{t t}-\mu \Delta v+v_{t}=0 & \text { in }(0, \infty) \times \mathbb{R}^{3}, \\
v(0, \cdot)=v_{0}, \quad v_{t}(0, \cdot)=v_{1} & \text { in } \mathbb{R}^{3} .
\end{array}
$$

Then for all $\alpha \in \mathbb{N}_{0}^{3}$ and $j \in \mathbb{N}_{0}$

$$
\begin{aligned}
& \left\|\nabla^{\alpha} \partial_{t}^{j} v(t, \cdot)\right\|_{2} \leq c(1+t)^{-\left(\frac{|\alpha|}{2}+j\right)}\left\|\left(v_{0}, v_{1}\right)\right\|_{X_{2}}, \\
& \left\|\nabla^{\alpha} \partial_{t}^{j} v(t, \cdot)\right\|_{2} \leq c(1+t)^{-\left(\frac{1}{4}+\frac{|\alpha|}{2}+j\right)}\left\|\left(v_{0}, v_{1}\right)\right\|_{X_{\frac{3}{2}}}, \\
& \left\|\nabla^{\alpha} \partial_{t}^{j} v(t, \cdot)\right\|_{2} \leq c(1+t)^{-\left(\frac{3}{4}+\frac{|\alpha|}{2}+j\right)}\left\|\left(v_{0}, v_{1}\right)\right\|_{X_{1}},
\end{aligned}
$$


with

$$
X_{k}:= \begin{cases}L^{2} \times L^{2} \cap L^{k} \times L^{k}, & \text { for }|\alpha|+j=0 \\ W^{|\alpha|+j, 2} \times W^{|\alpha|+j-1,2} \cap L^{k} \times L^{k}, & \text { for }|\alpha|+j \geq 1 .\end{cases}
$$

holds.

Proof. Use [8, Lemma 1] with $n=3$ and $m=2, m=\frac{3}{2}$ resp. $m=1$.

Now one successively deals with each term of $M(T)$. In contrast to the two dimensional case one always uses the following derivative structure of the nonlinearities. Because $\operatorname{div} u=0$, one can write

$$
\begin{aligned}
& N_{1}=-P((u \cdot \nabla) u)=-P(\nabla \cdot(u \otimes u)) \equiv P\left(\nabla \cdot \tilde{N}_{1}\right)=\nabla \cdot P \tilde{N}_{1} \\
& N_{2}=-P\left(\left(\tau u_{t} \cdot \nabla\right) u\right)=-P\left(\nabla \cdot\left(\tau u_{t} \otimes u\right)\right) \equiv P\left(\nabla \cdot \tilde{N}_{2}\right)=\nabla \cdot P \widetilde{N}_{2} \\
& N_{3}=-P\left((\tau u \cdot \nabla) u_{t}\right)=-P\left(\nabla \cdot\left(\tau u \otimes u_{t}\right)\right) \equiv P\left(\nabla \cdot \tilde{N}_{3}\right)=\nabla \cdot P \widetilde{N}_{3} .
\end{aligned}
$$

I. Estimate for $\|u(t)\|_{m, 2}$

Using the representation formula from Lemma 2.6

$u(t)=w(t)\left(u_{1}+\frac{1}{\tau} u_{0}\right)+\partial_{t} w(t) u_{0}+\frac{1}{\tau} \int_{0}^{t} w(t-r) \sum_{j=1}^{3} N_{j}(r) \mathrm{d} r \equiv v_{1}(t)+v_{2}(t)+v_{3}(t)$

one gets with (19) the estimates

$$
\left\|v_{1}(t)\right\|_{m, 2} \leq c(1+t)^{-\frac{3}{4}}\left(\left\|u_{0}\right\|_{m-1,2}+\left\|u_{1}\right\|_{m-1,2}+\left\|u_{0}\right\|_{1}+\left\|u_{1}\right\|_{1}\right)<c \delta_{1}(1+t)^{-\frac{3}{4}},
$$

$$
\left\|v_{2}(t)\right\|_{m, 2} \leq c(1+t)^{-\left(\frac{3}{4}+1\right)}\left(\left\|u_{0}\right\|_{m+1-1,2}+\left\|u_{0}\right\|_{1}\right)<c \delta_{1}(1+t)^{-\left(\frac{3}{4}+1\right)} .
$$

To estimate $v_{3}$ one uses the derivative structure of the nonlinearities and the continuity of the Helmholtz projection together with (18) and gets

$$
\left\|v_{3}(t)\right\|_{m, 2} \leq c \int_{0}^{t}(1+t-r)^{-\left(\frac{1}{4}+\frac{1}{2}\right)} \sum_{j=1}^{3}\left(\left\|\widetilde{N}_{j}(r)\right\|_{m+1-1,2}+\left\|\widetilde{N}_{j}(r)\right\|_{\frac{3}{2}}\right) \mathrm{d} r .
$$

REMARK 2.8. The concerning the convergence rate better estimate (19) cannot be used here, because the Helmholtz projection is not continuous on $L^{1}$. Otherwise the estimate (17) is not sufficient (this would avoid the $\|\cdot\|_{\frac{3}{2}}$ terms), since then the convergence rate of $\|u(t)\|_{m, 2}$ would be too small (compare (32)).

With Theorem 4.1 and the Sobolev embedding theorem $\left(m>\frac{3}{2}\right)$ it follows

$$
\begin{aligned}
\left\|\tilde{N}_{1}(r)\right\|_{m, 2} & \leq c\|u(r)\|_{\infty}\|u(r)\|_{m, 2} \\
& \leq c\|u(r)\|_{m, 2}\|u(r)\|_{m, 2} \\
& =c(1+r)^{-\frac{3}{4}}\left((1+r)^{\frac{3}{4}}\|u(r)\|_{m, 2}\right)(1+r)^{-\frac{3}{4}}\left((1+r)^{\frac{3}{4}}\|u(r)\|_{m, 2}\right) \\
& \leq c(1+r)^{-\frac{3}{2}} M(T)^{2}
\end{aligned}
$$


and

$$
\left\|\tilde{N}_{2}(r)\right\|_{m, 2}+\left\|\tilde{N}_{3}(r)\right\|_{m, 2} \leq c(1+r)^{-\frac{3}{4}-\frac{3}{2}} M(T)^{2}
$$

Because of $\frac{1}{\frac{3}{2}}=\frac{1}{2}+\frac{1}{6}$, one can use Hölder, which together with the Sobolev embedding theorem implies

$$
\left\|\tilde{N}_{1}(r)\right\|_{\frac{3}{2}} \leq c\|u(r)\|_{2}\|u(r)\|_{6} \leq c\|u(r)\|_{m, 2}\|u(r)\|_{m, 2} \leq c(1+r)^{-\frac{3}{2}} M(T)^{2}
$$

and

$$
\left\|\widetilde{N}_{2}(r)\right\|_{\frac{3}{2}}+\left\|\tilde{N}_{3}(r)\right\|_{\frac{3}{2}} \leq c(1+r)^{-\frac{3}{4}-\frac{3}{2}} M(T)^{2} .
$$

Plugging in all estimates for the $\widetilde{N}_{j}(r)$ in $(22)$, it follows

$$
\left\|v_{3}(t)\right\|_{m, 2} \leq c M(T)^{2}(1+t)^{-\frac{3}{4}} \int_{0}^{t}(1+t-r)^{-\left(\frac{1}{4}+\frac{1}{2}\right)}(1+r)^{-\frac{3}{2}}(1+t)^{\frac{3}{4}} \mathrm{~d} r .
$$

Using Lemma 4.2, one finally gets

$$
\left\|v_{3}(t)\right\|_{m, 2} \leq c M(T)^{2}(1+t)^{-\frac{3}{4}}
$$

Summing up (20), (21) and (24)

$$
(1+t)^{\frac{3}{4}}\|u(t)\|_{m, 2}<c \delta_{1}+c M(T)^{2}
$$

is shown.

II. Now to the estimate for $\left\|u_{t}(t)\right\|_{m, 2}$

Differentiating the representation formula from Lemma 2.6 with respect to $t$ yields

$$
\begin{aligned}
u_{t}(t) & =\partial_{t} w(t)\left(u_{1}+\frac{1}{\tau} u_{0}\right)+\partial_{t}^{2} w(t) u_{0}+\partial_{t} \frac{1}{\tau} \int_{0}^{t} w(t-r) \sum_{j=1}^{3} N_{j}(r) \mathrm{d} r \\
& =\partial_{t} w(t)\left(u_{1}+\frac{1}{\tau} u_{0}\right)+\partial_{t}^{2} w(t) u_{0}+\underbrace{\frac{1}{\tau} w(0) \sum_{j=1}^{3} N_{j}(t)}_{=0}+\frac{1}{\tau} \int_{0}^{t} \partial_{t} w(t-r) \sum_{j=1}^{3} N_{j}(r) \mathrm{d} r \\
& \equiv v_{1}(t)+v_{2}(t)+v_{3}(t)
\end{aligned}
$$

Analogous to I. it follows

$$
\begin{aligned}
\left\|v_{1}(t)\right\|_{m, 2} & \leq c(1+t)^{-\left(\frac{3}{4}+1\right)}\left(\left\|u_{0}\right\|_{m+1-1,2}+\left\|u_{1}\right\|_{m+1-1,2}+\left\|u_{0}\right\|_{1}+\left\|u_{1}\right\|_{1}\right) \\
& <c \delta_{1}(1+t)^{-\left(\frac{3}{4}+1\right)}, \\
\left\|v_{2}(t)\right\|_{m, 2} & \leq c(1+t)^{-\left(\frac{3}{4}+2\right)}\left(\left\|u_{0}\right\|_{m+2-1,2}+\left\|u_{0}\right\|_{1}\right)<c \delta_{1}(1+t)^{-\left(\frac{3}{4}+2\right)}
\end{aligned}
$$

Like above, but with (17) one gets

$$
\left\|v_{3}(t)\right\|_{m, 2} \leq c \int_{0}^{t}(1+t-r)^{-\left(\frac{1}{2}+1\right)} \sum_{j=1}^{3}\left\|\widetilde{N}_{j}(r)\right\|_{m+1+1-1,2} \mathrm{~d} r .
$$


Theorem 4.1 and the Sobolev embedding Theorem imply

$$
\left\|\widetilde{N}_{1}(r)\right\|_{m+1,2} \leq c\|u(r)\|_{m, 2}\|u(r)\|_{m+1,2}
$$

$\left\|\tilde{N}_{2}(r)\right\|_{m+1,2}+\left\|\tilde{N}_{3}(r)\right\|_{m+1,2} \leq c\left(\|u(r)\|_{m, 2}\left\|u_{t}(r)\right\|_{m+1,2}+\left\|u_{t}(r)\right\|_{m, 2}\|u(r)\|_{m+1,2}\right)$

$$
\leq c\|u(r)\|_{m, 2}\left(\|u(r)\|_{m, 2}+\left\|\nabla^{m+1} u(r)\right\|_{2}\right),
$$

$$
\begin{aligned}
& \leq c\left(\|u(r)\|_{m, 2}\left(\left\|u_{t}(r)\right\|_{m, 2}+\left\|\nabla^{m+1} u_{t}(r)\right\|_{2}\right)\right. \\
& \left.\quad+\left\|u_{t}(r)\right\|_{m, 2}\left(\|u(r)\|_{m, 2}+\left\|\nabla^{m+1} u(r)\right\|_{2}\right)\right) .
\end{aligned}
$$

The highest derivative can be estimated with the representation formula from Lemma 2.6 and (17) to

$$
\begin{aligned}
\left\|\nabla^{m+1} u(r)\right\|_{2} \leq & c(1+r)^{-\frac{m+1}{2}}\left\|u_{1}+\frac{1}{\tau} u_{0}\right\|_{m+1-1,2}+c(1+r)^{-\left(\frac{m+1}{2}+1\right)}\left\|u_{0}\right\|_{m+1+1-1,2} \\
& +c \int_{0}^{r}(1+r-\lambda)^{-\left(\frac{m+1}{2}+\frac{1}{2}\right)} \sum_{j=1}^{3}\left\|P \widetilde{N}_{j}(\lambda)\right\|_{m+1+1-1,2} \mathrm{~d} \lambda \\
\left\|\nabla^{m+1} u_{t}(r)\right\|_{2} \leq & c(1+r)^{-\left(\frac{m+1}{2}+1\right)}\left\|u_{1}+\frac{1}{\tau} u_{0}\right\|_{m+1+1-1,2}+c(1+r)^{-\left(\frac{m+1}{2}+2\right)}\left\|u_{0}\right\|_{m+1+2-1,2} \\
& +c \int_{0}^{r}(1+r-\lambda)^{-\left(\frac{m+1}{2}+\frac{1}{2}+1\right)} \sum_{j=1}^{3}\left\|P \widetilde{N}_{j}(\lambda)\right\|_{m+1+1+1-1,2} \mathrm{~d} \lambda .
\end{aligned}
$$

The high norm $\left\|P \widetilde{N}_{j}(\lambda)\right\|_{m+2,2}$ can be estimated by contiuity of the Helmholtz projection, Theorem 4.1 and the high energy estimate (Theorem 2.4) for $\bar{m}=m+1$ to

$$
\begin{aligned}
\left\|P \widetilde{N}_{j}(\lambda)\right\|_{m+2,2} & \leq c\left(\|u(\lambda)\|_{\infty}+\left\|u_{t}(\lambda)\right\|_{\infty}\right)\left(\|u(\lambda)\|_{m+2,2}+\left\|u_{t}(\lambda)\right\|_{m+2,2}\right) \\
& \leq c\left(\|u(\lambda)\|_{\infty}+\left\|u_{t}(\lambda)\right\|_{\infty}\right) \sqrt{E_{m+1}(\lambda)} \\
& \leq c\left(\|u(\lambda)\|_{\infty}+\left\|u_{t}(\lambda)\right\|_{\infty}\right) c \sqrt{E_{m+1}(0)} e^{c \int_{0}^{\lambda}\left(\|u\|_{\infty}^{2}+\left\|u_{t}\right\|_{1, \infty}+\|\nabla u\|_{\infty}\right)(\varrho) \mathrm{d} \varrho .}
\end{aligned}
$$

Using Sobolev $\left(m>\frac{3}{2}+1\right)$ and $\sqrt{E_{m+1}(0)} \leq c\left\|\left(u_{0}, u_{1}\right)\right\|_{W^{m+3,2} \times W^{m+2,2}}<c \delta_{1}$ it follows

(31) $\quad\left\|P \widetilde{N}_{j}(\lambda)\right\|_{m+2,2} \leq c \delta_{1}\left(\|u(\lambda)\|_{m, 2}+\left\|u_{t}(\lambda)\right\|_{m, 2}\right) e^{c \int_{0}^{\lambda}\left(\|u\|_{m, 2}^{2}+\left\|u_{t}\right\|_{m, 2}+\|\nabla u\|_{m, 2}\right)(\varrho) \mathrm{d} \varrho}$ and finally

$$
\begin{aligned}
& (1+r)^{\frac{3}{4}}\left(\left\|\nabla^{m+1} u(r)\right\|_{2}+\left\|\nabla^{m+1} u_{t}(r)\right\|_{2}\right) \\
& \leq c(1+r)^{-\frac{m+1}{2}+\frac{3}{4}}\left\|u_{1}+\frac{1}{\tau} u_{0}\right\|_{m+1,2}+c(1+r)^{-\left(\frac{m+1}{2}+1\right)+\frac{3}{4}}\left\|u_{0}\right\|_{m+2,2} \\
& +c \delta_{1} \int_{0}^{r}\left((1+r-\lambda)^{-\left(\frac{m+1}{2}+\frac{1}{2}\right)}(1+\lambda)^{-\frac{3}{4}}(1+r)^{\frac{3}{4}}\left[(1+\lambda)^{\frac{3}{4}}\|u(\lambda)\|_{m, 2}\right]\right. \\
& \left.+(1+r-\lambda)^{-\left(\frac{m+1}{2}+\frac{1}{2}\right)}(1+\lambda)^{-\frac{3}{2}}(1+r)^{\frac{3}{4}}\left[(1+\lambda)^{\frac{3}{2}}\left\|u_{t}(\lambda)\right\|_{m, 2}\right]\right) \\
& \quad e^{c \int_{0}^{\lambda}(1+\varrho)^{-2 \cdot \frac{3}{4}}\left[(1+\varrho)^{\frac{3}{4}}\|u(\varrho)\|_{m, 2}\right]^{2}+(1+\varrho)^{-\frac{5}{4}}\left[(1+\varrho)^{\frac{5}{4}}\left(\left\|u_{t}(\varrho)\right\|_{m, 2}+\|\nabla u(\varrho)\|_{m, 2}\right)\right] \mathrm{d} \varrho} \mathrm{d} \lambda .
\end{aligned}
$$

Lemma 4.2 yields

$$
\begin{aligned}
& \sup _{r \geq 0} \int_{0}^{r}(1+r-\lambda)^{-\left(\frac{m+1}{2}+\frac{1}{2}\right)}(1+\lambda)^{-\frac{3}{4}}(1+r)^{\frac{3}{4}} \mathrm{~d} \lambda<\infty, \\
& \sup _{r \geq 0} \int_{0}^{r}(1+r-\lambda)^{-\left(\frac{m+1}{2}+\frac{1}{2}\right)}(1+\lambda)^{-\frac{3}{2}}(1+r)^{\frac{3}{4}} \mathrm{~d} \lambda<\infty .
\end{aligned}
$$


Obviously it holds

$$
\sup _{\lambda \geq 0} \int_{0}^{\lambda}(1+\varrho)^{-\frac{3}{2}}+(1+\varrho)^{-\frac{5}{4}} \mathrm{~d} \varrho<\infty
$$

REMARK 2.9 (Comparison to the two dimensional case). This estimate is the essential difference between the two and the three dimensional case. In $\mathbb{R}^{2}$ it is not possible to estimate the norms in the exponent of e against $W^{m, 2}$-norms, because the integral (32) would be divergent, since the convergence rates in $\mathbb{R}^{2}$ are not good enough. Therefore in [12] $W^{m_{1}, q}$-estimates were used.

With these estimates and by definition of $\delta_{1}(14)$ and $M(T)(15)$ it follows

$$
(1+r)^{\frac{3}{4}}\left\|\nabla^{m+1} u(r)\right\|_{2}+\left\|\nabla^{m+1} u_{t}(r)\right\|_{2} \leq c \delta_{1}\left(1+M(T) e^{c\left(M(T)^{2}+M(T)\right)}\right) .
$$

Plugging in in (29) resp. (30) yields

$$
\begin{aligned}
& (1+r)^{\frac{3}{2}} \sum_{j=1}^{3}\left\|\widetilde{N}_{j}(r)\right\|_{m+1,2} \\
& \leq c(1+r)^{\frac{3}{4}}\|u(r)\|_{m, 2}\left((1+r)^{\frac{3}{4}}\|u(r)\|_{m, 2}+(1+r)^{\frac{3}{4}}\left\|\nabla^{m+1} u(r)\right\|_{2}\right) \\
& \quad+c\left((1+r)^{\frac{3}{4}}\|u(r)\|_{m, 2}\left((1+r)^{\frac{3}{4}}\left\|u_{t}(r)\right\|_{m, 2}+(1+r)^{\frac{3}{4}}\left\|\nabla^{m+1} u_{t}(r)\right\|_{m, 2}\right)\right. \\
& \left.\quad+(1+r)^{\frac{3}{4}}\left\|u_{t}(r)\right\|_{m, 2}\left((1+r)^{\frac{3}{4}}\|u(r)\|_{m, 2}+(1+r)^{\frac{3}{4}}\left\|\nabla^{m+1} u(r)\right\|_{m, 2}\right)\right) \\
& \leq c M(T)\left(M(T)+c \delta_{1}\left(1+M(T) e^{c\left(M(T)^{2}+M(T)\right)}\right)\right) .
\end{aligned}
$$

If one puts this in the estimate $(28)$ for $\left\|v_{3}(t)\right\|$, it follows

$$
\begin{aligned}
&\left\|v_{3}(t)\right\|_{m, 2} \leq c M(T)\left(M(T)+c \delta_{1}\left(1+M(T) e^{c\left(M(T)^{2}+M(T)\right)}\right)\right) \\
& \cdot(1+t)^{-\frac{3}{2}} \int_{0}^{t}(1+t-r)^{-\left(\frac{1}{2}+1\right)}(1+r)^{-\frac{3}{2}}(1+t)^{\frac{3}{2}} \mathrm{~d} r
\end{aligned}
$$

Again by an application of Lemma 4.2 one finally gets

(34) $(1+t)^{\frac{3}{2}}\left\|u_{t}(t)\right\|_{m, 2}<c \delta_{1}+c M(T)\left(M(T)+c \delta_{1}\left(1+M(T) e^{c\left(M(T)^{2}+M(T)\right)}\right)\right)$.

III. Estimate of $\|\nabla u(t)\|_{m, 2}$

Differentiating the representation formula from Lemma 2.6 with respect to $x$ yields

$$
\begin{aligned}
\nabla u(t) & =\nabla w(t)\left(u_{1}+\frac{1}{\tau} u_{0}\right)+\nabla \partial_{t} w(t) u_{0}+\frac{1}{\tau} \int_{0}^{t} \nabla w(t-r) \sum_{j=1}^{3} N_{j}(r) \mathrm{d} r \\
& \equiv v_{1}(t)+v_{2}(t)+v_{3}(t) .
\end{aligned}
$$


Like above it follows

$$
\begin{aligned}
\left\|v_{1}(t)\right\|_{m, 2} & \leq c(1+t)^{-\left(\frac{3}{4}+\frac{1}{2}\right)}\left(\left\|u_{0}\right\|_{m+1-1,2}+\left\|u_{1}\right\|_{m+1-1,2}+\left\|u_{0}\right\|_{1}+\left\|u_{1}\right\|_{1}\right) \\
& <c \delta_{1}(1+t)^{-\frac{5}{4}}, \\
\text { (35) } \quad\left\|v_{2}(t)\right\|_{m, 2} & \leq c(1+t)^{-\left(\frac{3}{4}+1+\frac{1}{2}\right)}\left(\left\|u_{0}\right\|_{m+1+1-1,2}+\left\|u_{0}\right\|_{1}\right)<c \delta_{1}(1+t)^{-\left(\frac{5}{4}+1\right)} .
\end{aligned}
$$

Like in I. one gets with (18) the estimate

$$
\left\|v_{3}(t)\right\|_{m, 2} \leq c \int_{0}^{t}(1+t-r)^{-\left(\frac{1}{4}+\frac{2}{2}\right)} \sum_{j=1}^{3}\left(\left\|\tilde{N}_{j}(r)\right\|_{m+2-1,2}+\left\|\widetilde{N}_{j}(r)\right\|_{\frac{3}{2}}\right) \mathrm{d} r .
$$

Now $\sum_{j=1}^{3}\left\|\widetilde{N}_{j}(r)\right\|_{m+1,2}$ can be estimated like in II., while $\sum_{j=1}^{3}\left(\left\|\widetilde{N}_{j}(r)\right\|_{\frac{3}{2}}\right.$ can be estimated like in I. such that

$$
\begin{aligned}
& (1+r)^{\frac{3}{2}} \sum_{j=1}^{3}\left(\left\|\widetilde{N}_{j}(r)\right\|_{m+1,2}+\left\|\widetilde{N}_{j}(r)\right\|_{\frac{3}{2}}\right) \\
& \quad \leq c M(T)\left(M(T)+c \delta_{1}\left(1+M(T) e^{c\left(M(T)^{2}+M(T)\right)}\right)\right)
\end{aligned}
$$

follows.

Plugging in in (37), Lemma 4.2 and (35) and (36) yield

(39) $(1+t)^{\frac{5}{4}}\|\nabla u(t)\|_{m, 2}<c \delta_{1}+c M(T)\left(M(T)+c \delta_{1}\left(1+M(T) e^{c\left(M(T)^{2}+M(T)\right)}\right)\right)$.

IV. Summary of I.-III. and proof of the Theorem

Putting together (25), (34) and (39) it is shown

$$
\begin{aligned}
& (1+t)^{\frac{3}{4}}\|u(t)\|_{m, 2}+(1+t)^{\frac{3}{2}}\left\|u_{t}(t)\right\|_{m, 2}+(1+t)^{\frac{5}{4}}\|\nabla u(t)\|_{m, 2} \\
& \quad<c \delta_{1}+c M(T)^{2}+c \delta_{1}+c M(T)\left(M(T)+c \delta_{1}\left(1+M(T) e^{c\left(M(T)^{2}+M(T)\right)}\right)\right) \\
& \leq c \delta_{1}+c M(T)^{2}+c \delta_{1} M(T)\left(1+M(T) e^{c\left(M(T)^{2}+M(T)\right)}\right) .
\end{aligned}
$$

By definition of $M(T)(15)$ one gets

$$
M(T) \leq c \delta_{1}+c M(T)^{2}+c \delta_{1} M(T)\left(1+M(T) e^{c\left(M(T)^{2}+M(T)\right)}\right)
$$

and therefore the claim follows by standard arguments (compare [12]).

Theorem 2.2 can now be followed as usual by applying the local existence Theorem 2.1 successively.

2.2. Half-space with slip boundary conditions. In this subsection the solvability of the hyperbolic Navier-Stokes equation in the half-space with slip boundary conditions is proven with a reflection technique. For simplicity we only deal with the three dimensional case. To be precise, the equation

(41)

$$
\begin{aligned}
\tau u_{t t}-\mu \Delta u & +u_{t}+\nabla p+\tau \nabla p_{t} \\
& =-(u \cdot \nabla) u-\left(\tau u_{t} \cdot \nabla\right) u-(\tau u \cdot \nabla) u_{t} \\
\operatorname{div} u & =0 \\
\frac{\partial u_{1}}{\partial \vec{n}}=0, \quad \frac{\partial u_{2}}{\partial \vec{n}} & =0, \quad u_{3}=0 \\
u(0, \cdot)=v, \quad u_{t}(0, \cdot)=w &
\end{aligned}
$$


for $u=u(t, x):(0, \infty) \times \mathbb{R}_{+}^{3} \rightarrow \mathbb{R}^{3}$ and $p=p(t, x):(0, \infty) \times \mathbb{R}_{+}^{3} \rightarrow \mathbb{R}$ with $\mu, \tau>0$ and given initial conditions $v$ and $w$ is treated.

Assuming for the initial conditions $v$ and $w$ for $m \in \mathbb{N}$ and $\delta>0$
$(\operatorname{V} 1(\boldsymbol{\delta}, \boldsymbol{m})) \quad(v, w) \in\left(W^{m+3,2}\left(\mathbb{R}_{+}^{3}\right) \cap L_{\sigma}^{2}\left(\mathbb{R}_{+}^{3}\right)\right) \times\left(W^{m+2,2}\left(\mathbb{R}_{+}^{3}\right) \cap L_{\sigma}^{2}\left(\mathbb{R}_{+}^{3}\right)\right)$ with $\|v\|_{m+3,2}+\|w\|_{m+2,2}+\|v\|_{1}+\|w\|_{1}<\delta$

$(\mathrm{V} 2(\boldsymbol{m})) \quad$ For all odd integers $k \in[3, m+2]$ the partial derivatives satisfy $\partial_{3}^{k} v_{1}, \partial_{3}^{k} v_{2} \in H_{0}^{1}\left(\mathbb{R}_{+}^{n}\right)$. Analogously for all odd integers $k \in$ $[3, m+1]$, it holds $\partial_{3}^{k} w_{1}, \partial_{3}^{k} w_{2} \in H_{0}^{1}\left(\mathbb{R}_{+}^{n}\right)\left(v_{j}\right.$ denotes the j-th component of the vector $v$ ).

Furthermore $v$ and $w$ satisfy the boundary condition.

the following Theorem can be proven.

Theorem 2.10 (Global solution in the half-space). Let $m \geq 3$, then there exists a $\delta>0$ such that if the initial conditions $v$ and $w$ satisfy $(V 1(\delta, m))$ and $(V 2(m))$ then there exists a unique global solution $(u, p)$ to the hyperbolic Navier-Stokes equation (41) with

$$
\begin{array}{r}
u \in C^{0}\left([0, \infty), W^{m+3,2}\left(\mathbb{R}_{+}^{3}\right) \cap L_{\sigma}^{2}\left(\mathbb{R}_{+}^{3}\right)\right) \\
\cap C^{1}\left([0, \infty), W^{m+2,2}\left(\mathbb{R}_{+}^{3}\right)\right) \\
\cap C^{2}\left([0, \infty), W^{m+1,2}\left(\mathbb{R}_{+}^{3}\right)\right), \\
\nabla\left(p+\tau p_{t}\right) \in C^{0}\left([0, \infty), W^{m+1,2}\left(\mathbb{R}_{+}^{3}\right)\right)
\end{array}
$$

Furthermore

$$
\begin{aligned}
\|u(t)\|_{m, 2}=\mathcal{O}\left(t^{-\frac{3}{4}}\right), & \left\|u_{t}(t)\right\|_{m, 2}=\mathcal{O}\left(t^{-\frac{3}{2}}\right), \\
\|\nabla u(t)\|_{m, 2}=\mathcal{O}\left(t^{-\frac{5}{4}}\right) & \text { for } t \rightarrow \infty
\end{aligned}
$$

holds.

Hints on the proof. The basic idea is to reflect the initial data to functions in the whole space, then apply the Theorem 2.2 for the whole space and finally check that the restriction of this solution to the half-space solves (41). In [15] this idea was described in a general context and applied for the classical Navier-Stokes equation. Therefore one actually applies Theorems from [15].

The boundary conditions dictate how to reflect the initial conditions, which means even extension of the first and second component of the vector and odd extension of the last component.

$$
\tilde{f}\left(x_{1}, x_{2}, x_{3}\right):=\left\{\begin{array}{ll}
\left(\begin{array}{c}
f_{1}\left(x_{1}, x_{2}, x_{3}\right) \\
f_{2}\left(x_{1}, x_{2}, x_{3}\right) \\
f_{3}\left(x_{1}, x_{2}, x_{3}\right) \\
f_{1}\left(x_{1}, x_{2},-x_{3}\right) \\
f_{2}\left(x_{1}, x_{2},-x_{3}\right) \\
-f_{3}\left(x_{1}, x_{2},-x_{3}\right)
\end{array}\right), & \text { for } x_{3} \geq 0,
\end{array} \quad \text { for } x_{3}<0 \quad f \in\{v, w\} .\right.
$$

The assumption $(V 2(m))$ guarantees thanks to [15, Theorem 5.1] that this extension is smooth, which means

$$
(\widetilde{v}, \widetilde{w}) \in\left(W^{m+3,2}\left(\mathbb{R}^{3}\right) \cap L_{\sigma}^{2}\left(\mathbb{R}^{3}\right)\right) \times\left(W^{m+2,2}\left(\mathbb{R}^{3}\right) \cap L_{\sigma}^{2}\left(\mathbb{R}^{3}\right)\right) .
$$


Choosing $\delta<\frac{\bar{\delta}}{2}$ with $\bar{\delta}$ from Theorem 2.2 the assumption $(\mathrm{V} 1(\delta, m))$ ensures that Theorem 2.2 can be applied to the initial data $(\widetilde{v}, \widetilde{w})$ which yields a solution $(\widetilde{u}, \widetilde{p})$ to the whole space problem.

Now one only has to check that

$$
(u, p):=\left(\left.\widetilde{u}\right|_{\mathbb{R}_{+}^{3}},\left.\widetilde{p}\right|_{\mathbb{R}_{+}^{3}}\right)
$$

has the right boundary conditions. But this is clear because the equation preserves the symmetry of the initial conditions $(\widetilde{v}, \widetilde{w})$ (compare $[15$, Proposition 4.1]).

The claimed convergence rates follow by construction of the solution directly from Theorem 2.2. The uniqueness of the solution can be proven with the multiplier method (compare Section 3.2).

3. Relaxation parameter $\tau \rightarrow 0$. Looking at the derivation of the hyperbolic Navier-Stokes equation one would expect that for vanishing relaxation parameter $\tau$ the corresponding solutions $u^{\tau}$ converge to the solution $v$ of the classical Navier-Stokes equation, but as mentioned in the introduction this is not clear.

It will be shown that in the whole space $\mathbb{R}^{n}$ with $n=2$ or $n=3$ for sufficiently smooth initial data, $T_{1}>0$ appropiate and $m>\frac{n}{2}$ (48)

$$
\sup _{0 \leq t \leq T_{1}}\left\|u^{\tau}(t)-v(t)\right\|_{m+2,2}=\mathcal{O}(\tau) \quad \text { and } \quad \sup _{0 \leq t \leq T_{1}}\left\|u_{t}^{\tau}(t)-v_{t}(t)\right\|_{m+1,2}=\mathcal{O}(\sqrt{\tau})
$$

for $\tau \rightarrow 0$ holds.

This will be proven with the multiplier method applied to an equation for the difference $w:=u^{\tau}-v$. Therefore one first needs an appropriate equation for $w$.

Differentiating the Helmholtz-projected Navier-Stokes equation for $v \in L_{\sigma}^{2}\left(\mathbb{R}^{n}\right)$ with respect to $t$ yields

$$
\begin{aligned}
v_{t}-\mu \Delta v+P((v \cdot \nabla) v) & =0 & \text { in }(0, T) \times \mathbb{R}^{n}, \\
v(0, \cdot) & =v_{0} & \text { in } \mathbb{R}^{n} .
\end{aligned}
$$

This equation is now multiplied with $\tau$ and then added to the original equation and hence

$$
\begin{array}{rlr}
\tau v_{t t}-\mu \Delta v & -\tau \mu \Delta v_{t}+v_{t} & \\
= & -P((v \cdot \nabla) v)-P\left(\left(\tau v_{t} \cdot \nabla\right) v\right) & \\
& -P\left((\tau v \cdot \nabla) v_{t}\right) & \\
v(0, \cdot)=v_{0}, \quad & v_{t}(0, \cdot)=\mu \Delta v_{0}-P\left(\left(v_{0} \cdot \nabla\right) v_{0}\right)=: v_{1} & \text { in }(0, T) \times \mathbb{R}^{n},
\end{array}
$$

The initial condition for $v_{t}(0, \cdot)$ is a natural compability condition from (49). Subtraction of this equation from the Helmholtz-projected hyperbolic Navier-Stokes equation for $u \in L_{\sigma}^{2}\left(\mathbb{R}^{n}\right)$

$$
\begin{array}{ccc}
\tau u_{t t}-\mu \Delta u+u_{t}= & -P((u \cdot \nabla) u)-P\left(\left(\tau u_{t} \cdot \nabla\right) u\right) & \\
& -P\left((\tau u \cdot \nabla) u_{t}\right) \equiv N_{1}+N_{2}+N_{3} & \text { in }(0, T) \times \mathbb{R}^{n}, \\
u(0, \cdot)=v_{0}, \quad u_{t}(0, \cdot)=v_{1} & \text { in } \mathbb{R}^{n},
\end{array}
$$

gives the desired equation for $L_{\sigma}^{2}\left(\mathbb{R}^{n}\right) \ni w:=u-v$

$$
\begin{array}{cl}
\tau w_{t t}-\mu \Delta w+w_{t}+\tau \mu \Delta v_{t}=\sum_{j=1}^{6} M_{j} & \text { in }(0, T) \times \mathbb{R}^{n}, \\
w(0, \cdot)=0, \quad w_{t}(0, \cdot)=0 & \text { in } \mathbb{R}^{n} .
\end{array}
$$


Where the $M_{j}$ are determined by

$$
\begin{aligned}
& -P(u \cdot \nabla) u+P(v \cdot \nabla) v=-P(u \cdot \nabla)(u-v)-P(u \cdot \nabla) v+P(v \cdot \nabla) v \\
= & -P(u \cdot \nabla)(u-v)-P((u-v) \cdot \nabla) v=-P(u \cdot \nabla) w-P(w \cdot \nabla) v \equiv M_{1}+M_{2},
\end{aligned}
$$

resp.

$$
\begin{aligned}
& -\tau P\left(u_{t} \cdot \nabla\right) u-\tau P(u \cdot \nabla) u_{t}+\tau P\left(v_{t} \cdot \nabla\right) v+\tau P(v \cdot \nabla) v_{t} \\
= & -\tau P\left(u_{t} \cdot \nabla\right) w-\tau P\left(w_{t} \cdot \nabla\right) v-\tau P(u \cdot \nabla) w_{t}-\tau P(w \cdot \nabla) v_{t} \\
\equiv & M_{3}+M_{4}+M_{5}+M_{6} .
\end{aligned}
$$

The following energy estimate is similar to the methods used to proof the high energy estimate [12, Theorem 4.1], but first we have to specify the regularity of the solutions $u$ and $v$. Actually the solutions should be just as good as that the following calculation works, which can surely be achieved if $v_{0}$ is smooth enough and $m>\frac{n}{2}$. To be precise Theorem 2.1 gives a solution

$$
\begin{aligned}
u^{\tau} \in C^{0}\left(\left[0, T^{\tau}\right], W^{m+3,2}\left(\mathbb{R}^{n}\right) \cap L_{\sigma}^{2}\left(\mathbb{R}^{n}\right)\right) & \cap C^{1}\left(\left[0, T^{\tau}\right], W^{m+2,2}\left(\mathbb{R}^{n}\right)\right) \\
& \cap C^{2}\left(\left[0, T^{\tau}\right], W^{m+1,2}\left(\mathbb{R}^{n}\right)\right)
\end{aligned}
$$

to the hyperbolic Navier-Stokes equation and for example with [14] one can derive a solution

$$
v \in C^{1}\left(\left[0, T_{v}\right], W^{m+3,2}\left(\mathbb{R}^{n}\right) \cap L_{\sigma}^{2}\left(\mathbb{R}^{n}\right)\right) \cap C^{2}\left(\left[0, T_{v}\right], W^{m+1,2}\left(\mathbb{R}^{n}\right)\right)
$$

to the classical Navier-Stokes equation.

It will be shown that $T_{1}>0$ can be chosen independent from $\tau$ such that all solutions exist on a common interval $\left[0, T_{1}\right]$. Therefore the following definition and the subsequent calculation makes sense for $t \in\left[0, T_{1}\right]$.

One defines the energies by

$$
E_{m}(\varphi(t)):=\frac{1}{2} \sum_{|\alpha| \leq m+1}\left(\tau\left\|\nabla^{\alpha} \varphi_{t}\right\|_{2}^{2}+\mu\left\|\nabla^{\alpha} \nabla \varphi\right\|_{2}^{2}+\left\|\nabla^{\alpha} \varphi\right\|_{2}^{2}\right)(t) \quad \text { for } \varphi \in\{u, v, w\}
$$

and corresponding Lyapunov functionals by

$$
\widetilde{E}_{m}(\varphi(t)):=E_{m}(\varphi(t))+\tau \sum_{|\alpha| \leq m+1}\left\langle\nabla^{\alpha} \varphi_{t}, \nabla^{\alpha} \varphi\right\rangle(t) \quad \text { for } \varphi \in\{u, w\} .
$$

For $\tau \leq \frac{1}{2}$ it holds

$$
\frac{1}{4} E_{m}(\varphi(t)) \leq \widetilde{E}_{m}(\varphi(t)) \leq \frac{7}{4} E_{m}(\varphi(t))
$$

In subsection 3.2 it will be shown that for $R>0$ appropriate and $\tau$ small enough the estimate

(58)

$$
\begin{aligned}
& E_{m}(w(t)) \\
& \leq \int_{0}^{t} c \tau^{2} R \mathrm{~d} r \cdot \exp (c \int_{0}^{t} \underbrace{\left(E_{m}(u(r))+E_{m+1}(v(r))+E_{m}(u(r))^{\frac{1}{2}}+E_{m+1}(v(r))^{\frac{1}{2}}+1\right)}_{=: E(r)} \mathrm{d} r)
\end{aligned}
$$


holds, where $c$ is a constant independent from $\tau$ (this shall hold for the whole section). Since in subsection 3.1 for $T_{1}>0$ appropriate the boundedness of $E_{m}(u(t))$ in $\tau$ for $t \in\left[0, T_{1}\right]$ is shown, $E(t)$ stays bounded in $\tau$ for $t \in\left[0, T_{1}\right]$ and the following Theorem is proven. that

THEOREM 3.1. For $m>\frac{n}{2}$ and $v_{0}$ sufficiently smooth there exists a $T_{1}>0$ such

$$
\sup _{0 \leq t \leq T_{1}} E_{m}(w(t))=\mathcal{O}\left(\tau^{2}\right) \quad \text { for } \tau \rightarrow 0
$$

Especially

(60)

$$
\sup _{0 \leq t \leq T_{1}}\left\|u^{\tau}(t)-v(t)\right\|_{m+2,2}=\mathcal{O}(\tau) \quad \text { and } \quad \sup _{0 \leq t \leq T_{1}}\left\|u_{t}^{\tau}(t)-v_{t}(t)\right\|_{m+1,2}=\mathcal{O}(\sqrt{\tau})
$$

holds. This means that the solutions $u^{\tau}$ of the hyperbolic Navier-Stokes equation converge for $\tau \rightarrow 0$ to the solution $v$ of the classical Navier-Stokes equation.

\subsection{Boundedness of $u^{\tau}$ in $\tau$.}

THEOREM 3.2. There exists a $T_{1}>0$ such that for $m>\frac{n}{2}$ and $v_{0} \in W^{m+4,2}\left(\mathbb{R}^{n}\right)$

$$
\sup _{0 \leq t \leq T_{1}} E_{m}(u(t))=\mathcal{O}(1) \quad \text { for } \tau \rightarrow 0 .
$$

Proof. Under the assumption that the solutions $\left(u^{\tau}\right)_{\tau}$ exist on a common interval $[0, T]$ with $T$ independent from $\tau$, for small $\tau$ the a priori estimate

$$
\widetilde{E}_{m}(u(t)) \leq \widetilde{E}_{m}(u(0))+c \int_{0}^{t}\left(\widetilde{E}_{m}(u(r))+1\right)^{2} \mathrm{~d} r,
$$

will be shown. Then one can apply a nonlinear version of the Lemma from Gronwall 4.3 and gets the claim.

For reasons of clarity the argument $(t)$ is dropped. yields

Let $|\alpha| \leq m+1$. Applying $\nabla^{\alpha}$ to $(51)$ and multiplication with $\nabla^{\alpha} u_{t}$ in $L^{2}\left(\mathbb{R}^{n}\right)$

$$
\frac{\tau}{2} \frac{\mathrm{d}}{\mathrm{d} t}\left\|\nabla^{\alpha} u_{t}\right\|_{2}^{2}+\frac{\mu}{2} \frac{\mathrm{d}}{\mathrm{d} t}\left\|\nabla^{\alpha} \nabla u\right\|_{2}^{2}+\left\|\nabla^{\alpha} u_{t}\right\|_{2}^{2}=\sum_{j=1}^{3}\left\langle\nabla^{\alpha} N_{j}, \nabla^{\alpha} u_{t}\right\rangle
$$

Now one has to estimate the nonlinear terms. In doing so, one has to observe that terms like $\left\|\nabla^{\alpha} u_{t}\right\|_{2}^{2}$ can only be estimated $\tau$-independent against $E_{m}(u)$ if they have a prefactor $\tau$. If $\tau$ appears in front of other terms like $\tau\left\|\nabla^{\alpha} u\right\|_{2}^{2}$, one can estimate them $\tau$-independent, if one assumes without restriction $\tau<1$.

With the continuity of the Helmholtz projection and Theorem 4.1 one gets

$$
\left|\left\langle\nabla^{\alpha} N_{1}, \nabla^{\alpha} u_{t}\right\rangle\right| \leq c\left(\|u\|_{\infty}\left\|\nabla^{m+1} \nabla u\right\|_{2}+\|\nabla u\|_{\infty}\left\|\nabla^{m+1} u\right\|_{2}\right)\left\|\nabla^{\alpha} u_{t}\right\|_{2} .
$$

Using Young's inequality and Sobolev yields

$$
\begin{aligned}
\left|\left\langle\nabla^{\alpha} N_{1}, \nabla^{\alpha} u_{t}\right\rangle\right| & \leq c\left(\|u\|_{m, 2}^{2}\left\|\nabla^{m+1} \nabla u\right\|_{2}^{2}+\|\nabla u\|_{m, 2}^{2}\left\|\nabla^{m+1} u\right\|_{2}^{2}\right)+\frac{1}{2}\left\|\nabla^{\alpha} u_{t}\right\|_{2}^{2} \\
& \leq c E_{m}(u)^{2}+\frac{1}{2}\left\|\nabla^{\alpha} u_{t}\right\|_{2}^{2} .
\end{aligned}
$$


Analogously

$$
\begin{aligned}
\left|\left\langle\nabla^{\alpha} N_{2}, \nabla^{\alpha} u_{t}\right\rangle\right| & \leq c \tau\left(\left\|u_{t}\right\|_{m, 2}^{2}\left\|\nabla^{m+1} \nabla u\right\|_{2}^{2}+\|\nabla u\|_{m, 2}^{2}\left\|\nabla^{m+1} u_{t}\right\|_{2}^{2}\right)+c \tau\left\|\nabla^{\alpha} u_{t}\right\|_{2}^{2} \\
& \leq c E_{m}(u)^{2}+c\left(E_{m}(u)+1\right)^{2} .
\end{aligned}
$$

The last term can be rearranged to

$$
\begin{aligned}
\left\langle\nabla^{\alpha} N_{3}, \nabla^{\alpha} u_{t}\right\rangle & =-\tau\left\langle\left(u \cdot \nabla \nabla^{\alpha}\right) u_{t}, \nabla^{\alpha} u_{t}\right\rangle-\tau\left\langle\nabla^{\alpha}\left((u \cdot \nabla) u_{t}\right)-\left(u \cdot \nabla^{\alpha} \nabla\right) u_{t}, \nabla^{\alpha} u_{t}\right\rangle \\
& \equiv R_{1}+R_{2} .
\end{aligned}
$$

With integration by parts one gets

$$
\begin{aligned}
R_{1} & =-\tau\left\langle u_{j} \partial_{j} \partial^{\alpha} \partial_{t} u_{r}, \partial^{\alpha} \partial_{t} u_{r}\right\rangle \stackrel{\operatorname{div} \underline{=}=0}{=}-\tau\left\langle\partial_{j} u_{j} \partial^{\alpha} \partial_{t} u_{r}, \partial^{\alpha} \partial_{t} u_{r}\right\rangle \\
& =\tau\left\langle u_{j} \partial^{\alpha} \partial_{t} u_{r}, \partial_{j} \partial^{\alpha} \partial_{t} u_{r}\right\rangle=\tau\left\langle\partial^{\alpha} \partial_{t} u_{r}, u_{j} \partial_{j} \partial^{\alpha} \partial_{t} u_{r}\right\rangle=-R_{1}
\end{aligned}
$$

and therefore $R_{1}=0$.

The term $R_{2}$ can be estimated with Theorem 4.1 (ii) to

$$
\begin{aligned}
\left|R_{2}\right| & \leq c \tau\left\|\nabla^{\alpha}\left((u \cdot \nabla) u_{t}\right)-\left(u \cdot \nabla^{\alpha} \nabla\right) u_{t}\right\|_{2}\left\|\nabla^{\alpha} u_{t}\right\|_{2} \\
& \leq c \tau\left(\|\nabla u\|_{\infty}\left\|\nabla^{m} \nabla u_{t}\right\|_{2}+\left\|\nabla u_{t}\right\|_{\infty}\left\|\nabla^{m+1} u\right\|_{2}\right)\left\|\nabla^{\alpha} u_{t}\right\|_{2} \\
& \leq c \tau\left(\|\nabla u\|_{m, 2}^{2}\left\|\nabla^{m} \nabla u_{t}\right\|_{2}^{2}+\left\|\nabla u_{t}\right\|_{m, 2}^{2}\left\|\nabla^{m+1} u\right\|_{2}^{2}\right)+c \tau\left\|\nabla^{\alpha} u_{t}\right\|_{2}^{2} \\
& \leq c E_{m}(u)^{2}+c\left(E_{m}(u)+1\right)^{2} .
\end{aligned}
$$

Now one again applies for all $|\alpha| \leq m+1$ the operator $\nabla^{\alpha}$ to (51) but this time multiplies with $\nabla^{\alpha} u$ in $L^{2}\left(\mathbb{R}^{n}\right)$ to the end

(68) $\tau \frac{\mathrm{d}}{\mathrm{d} t}\left\langle\nabla^{\alpha} u_{t}, \nabla^{\alpha} u\right\rangle-\tau\left\|\nabla^{\alpha} u_{t}\right\|_{2}^{2}+\mu\left\|\nabla^{\alpha} \nabla u\right\|_{2}^{2}+\frac{\mathrm{d}}{\mathrm{d} t} \frac{1}{2}\left\|\nabla^{\alpha} u\right\|_{2}^{2}=\sum_{j=1}^{3}\left\langle\nabla^{\alpha} N_{j}, \nabla^{\alpha} u\right\rangle$.

As above one gets

$$
\left|\left\langle\nabla^{\alpha} N_{1}, \nabla^{\alpha} u\right\rangle\right|+\left|\left\langle\nabla^{\alpha} N_{2}, \nabla^{\alpha} u\right\rangle\right| \leq c E_{m}(u)^{2}+c\left(E_{m}(u)+1\right)^{2} .
$$

For the last term one distinguishes between $|\alpha|=0$ and $|\alpha| \neq 0$. For $|\alpha|=0$

$$
\begin{aligned}
\left|\left\langle N_{3}, u\right\rangle\right| & \leq c \tau\left\|(u \cdot \nabla) u_{t}\right\|_{2}\|u\|_{2} \leq c \tau\left\|\nabla u_{t}\right\|_{\infty}\|u\|_{2}^{2} \\
& \leq c \tau\left\|\nabla u_{t}\right\|_{m, 2}^{2}+c \tau\|u\|_{2}^{4} \leq c\left(E_{m}(u)+1\right)^{2}+c E_{m}(u)^{2}
\end{aligned}
$$

holds, while for $|\alpha| \neq 0$ there exists a $1 \leq k \leq n$ with $\alpha_{k} \neq 0$ and therefore

$$
\begin{aligned}
\left|\left\langle\nabla^{\alpha} N_{3}, \nabla^{\alpha} u\right\rangle\right| & \leq c \tau\left(\|u\|_{\infty}\left\|\nabla^{m} \nabla u_{t}\right\|_{2}+\left\|\nabla u_{t}\right\|_{\infty}\left\|\nabla^{m} u\right\|_{2}\right)\left\|\nabla^{\alpha+e_{k}} u\right\|_{2} \\
& \leq c \tau\left(\|u\|_{m, 2}^{2}\left\|\nabla^{m} \nabla u_{t}\right\|_{2}^{2}+\left\|\nabla u_{t}\right\|_{m, 2}^{2}\left\|\nabla^{m} u\right\|_{2}^{2}\right)+c \tau\left\|\nabla^{\alpha+e_{k}} u\right\|_{2}^{2} \\
& \leq c E_{m}(u)^{2}+c\left(E_{m}(u)+1\right)^{2} .
\end{aligned}
$$

Adding (63) and (68), summing up over $|\alpha| \leq m+1$ and using the estimates for the nonlinearities $(65),(66),(67)$ and $(69),(70)$ resp. (71) yields

$$
\begin{aligned}
\frac{\mathrm{d}}{\mathrm{d} t} \widetilde{E}_{m}(u)+ & \sum_{|\alpha| \leq m+1}\left(\left\|\nabla^{\alpha} u_{t}\right\|_{2}^{2}-\tau\left\|\nabla^{\alpha} u_{t}\right\|_{2}^{2}+\mu\left\|\nabla^{\alpha} \nabla u\right\|_{2}^{2}\right) \\
& \leq c E_{m}(u)^{2}+c\left(E_{m}(u)+1\right)^{2}+\sum_{|\alpha| \leq m+1} \frac{1}{2}\left\|\nabla^{\alpha} u_{t}\right\|_{2}^{2}
\end{aligned}
$$


Without loss of generality one sets $\tau \leq \frac{1}{2}$ and obtains

$$
\frac{\mathrm{d}}{\mathrm{d} t} \widetilde{E}_{m}(u) \leq c E_{m}(u)^{2}+c\left(E_{m}(u)+1\right)^{2} \leq c\left(E_{m}(u)+1\right)^{2} .
$$

If one integrates in time from 0 to $t$ and uses (57), the estimate (62) is proven. To apply the nonlinear version of the Lemma from Gronwall 4.3 one has to look at the differential equation

$$
\begin{aligned}
\dot{h}(t) & =c(h(t)+1)^{2} \\
h(0) & =\frac{7}{4} E_{m}(u(0))=: h_{0},
\end{aligned}
$$

which clearly has the solution

$$
h(t)=\frac{h_{0}+1}{1-t c\left(h_{0}+1\right)}-1 \quad \text { for } t \in\left[0, \frac{1}{c\left(h_{0}+1\right)}\right) .
$$

The existence interval of $h$ gets larger for $\tau \rightarrow 0$. Setting $\delta$ equal to the value of $\frac{7}{4} E_{m}(u(0))$ for $\tau=\frac{1}{2}$ and $T_{1}$ as $0<T_{1}<\frac{1}{c(\delta+1)}$, then the continuous function $h=h(t, \tau):\left[0, T_{1}\right] \times\left[0, \frac{1}{2}\right] \rightarrow \mathbb{R}$ has a maximum $M$ and therefore

$$
\sup _{0 \leq t \leq T_{1}} E_{m}(u(t)) \leq \sup _{0 \leq t \leq T_{1}} h(t) \leq M \quad \text { for } \tau \in\left[0, \frac{1}{2}\right]
$$

It remains to show, that $\left(u^{\tau}\right)_{\tau}$ for $\tau \in\left[0, \frac{1}{2}\right]$ arbitrary exist on the common interval $\left[0, T_{1}\right]$.

Let $\tau \in\left[0, \frac{1}{2}\right]$. Then $u^{\tau}$ exists on $\left[0, T^{\tau}\right]$, where $T^{\tau}=T^{\tau}\left(\left\|u_{0}\right\|_{m+2},\left\|u_{1}\right\|_{m+1}, \tau\right)$. If $T^{\tau} \geq T_{1}$ nothing is to prove and therefore let $T^{\tau}<T_{1}$. From the estimate above one gets $E_{m}\left(u^{\tau}\left(T^{\tau}\right)\right) \leq M$. Now the local existence theorem can be applied to the initial data $u^{\tau}\left(T^{\tau}\right)$ and $u_{t}^{\tau}\left(T^{\tau}\right)$ and therefore one gets a solution on $\left[T^{\tau}, \widetilde{T}^{\tau}\right]$ with $\widetilde{T}^{\tau}=\widetilde{T}^{\tau}\left(M, \frac{M}{\tau}, \tau\right)$. If $\widetilde{T}^{\tau}<T_{1}$ again $E_{m}\left(u^{\tau}\left(\widetilde{T}^{\tau}\right)\right) \leq M$ holds and hence one can argument successively.

REMARK 3.3 (Dependences on $T_{1}$ and $M$ ). $T_{1}$ is defined by the existence time of $h$ (cp. (73)) and therefore depends on the norm of the initial data and on the constant $c$ that comes from the estimate above. To be more precise $c$ depends on the constant coming from the Moser inequalities, on $\mu$ and so on but most important $c$ and $T_{1}$ do not depend on $\tau$. $M$ is by definition the maximum of $h$ and so depends on the same quantities as $c$ and $T_{1}$.

3.2. Convergence for $\tau \rightarrow 0$. Now we want to proof Theorem 3.1.

The estimate is very similiar to the one of the previous subsection, especially one again has to observe that terms with time derivative must have a prefactor $\tau$ to estimate them against the energy. Of course for terms like $\left\|v_{t}\right\|_{m, 2}$ this is not necessary, but for clarity and uniformity we do so. Without restriction let $T_{1} \leq T_{v}$.

For a suitable $R>0$ and $\tau$ small enough we prove with the multiplier method the estimate

$$
\begin{aligned}
& \frac{\mathrm{d}}{\mathrm{d} t} \widetilde{E}_{m}(w(t)) \\
& \leq c \tau^{2} R+c\left(E_{m}(u(t))+E_{m+1}(v(t))+E_{m}(u(t))^{\frac{1}{2}}+E_{m+1}(v(t))^{\frac{1}{2}}+1\right) \widetilde{E}_{m}(w(t)),
\end{aligned}
$$


from which one easily obtains (58) and the claim.

As above one gets

(76)

$\frac{\tau}{2} \frac{\mathrm{d}}{\mathrm{d} t}\left\|\nabla^{\alpha} w_{t}\right\|_{2}^{2}+\frac{\mu}{2} \frac{\mathrm{d}}{\mathrm{d} t}\left\|\nabla^{\alpha} \nabla w\right\|_{2}^{2}+\left\|\nabla^{\alpha} w_{t}\right\|_{2}^{2}=-\left\langle\mu \tau \nabla^{\alpha} \Delta v_{t}, \nabla^{\alpha} w_{t}\right\rangle+\sum_{j=1}^{6}\left\langle\nabla^{\alpha} M_{j}, \nabla^{\alpha} w_{t}\right\rangle$.

Without restriction let again $\tau<1$ hold. Analogously to the previous section one gets

$$
\begin{aligned}
\left|\left\langle\nabla^{\alpha} M_{1}, \nabla^{\alpha} w_{t}\right\rangle\right| & \leq c\left(\|u\|_{\infty}\left\|\nabla^{m+1} \nabla w\right\|_{2}+\|\nabla w\|_{\infty}\left\|\nabla^{m+1} u\right\|_{2}\right)\left\|\nabla^{\alpha} w_{t}\right\|_{2} \\
& \leq c\left(\|u\|_{m, 2}^{2}+\left\|\nabla^{m+1} u\right\|_{2}^{2}\right)\left(\left\|\nabla^{m+1} \nabla w\right\|_{2}^{2}+\|\nabla w\|_{m, 2}^{2}\right)+\frac{1}{6}\left\|\nabla^{\alpha} w_{t}\right\|_{2}^{2} \\
& \leq c E_{m}(u) E_{m}(w)+\frac{1}{6}\left\|\nabla^{\alpha} w_{t}\right\|_{2}^{2}
\end{aligned}
$$

and

$$
\begin{aligned}
\left|\left\langle\nabla^{\alpha} M_{2}, \nabla^{\alpha} w_{t}\right\rangle\right| & \leq c\left(\|w\|_{\infty}\left\|\nabla^{m+1} \nabla v\right\|_{2}+\|\nabla v\|_{\infty}\left\|\nabla^{m+1} w\right\|_{2}\right)\left\|\nabla^{\alpha} w_{t}\right\|_{2} \\
& \leq c E_{m}(v) E_{m}(w)+\frac{1}{6}\left\|\nabla^{\alpha} w_{t}\right\|_{2}^{2} .
\end{aligned}
$$

Furthermore it holds

$$
\begin{aligned}
\left|\left\langle\nabla^{\alpha} M_{3}, \nabla^{\alpha} w_{t}\right\rangle\right| & \leq c \tau\left(\left\|u_{t}\right\|_{\infty}\left\|\nabla^{m+1} \nabla w\right\|_{2}+\|\nabla w\|_{\infty}\left\|\nabla^{m+1} u_{t}\right\|_{2}\right)\left\|\nabla^{\alpha} w_{t}\right\|_{2} \\
& \leq c \tau^{2}\left(\left\|u_{t}\right\|_{m, 2}^{2}+\left\|\nabla^{m+1} u_{t}\right\|_{2}^{2}\right)\left(\left\|\nabla^{m+1} \nabla w\right\|_{2}^{2}+\|\nabla w\|_{m, 2}^{2}\right)+\frac{1}{6}\left\|\nabla^{\alpha} w_{t}\right\|_{2}^{2} \\
& \leq c \tau E_{m}(u) E_{m}(w)+\frac{1}{6}\left\|\nabla^{\alpha} w_{t}\right\|_{2}^{2} .
\end{aligned}
$$

and

$$
\begin{aligned}
\left|\left\langle\nabla^{\alpha} M_{4}, \nabla^{\alpha} w_{t}\right\rangle\right| & \leq c \tau\left(\left\|w_{t}\right\|_{\infty}\left\|\nabla^{m+1} \nabla v\right\|_{2}+\|\nabla v\|_{\infty}\left\|\nabla^{m+1} w_{t}\right\|_{2}\right)\left\|\nabla^{\alpha} w_{t}\right\|_{2} \\
& \leq c \tau\left(\left\|\nabla^{m+1} \nabla v\right\|_{2}+\|\nabla v\|_{\infty}\right)\left(\left\|w_{t}\right\|_{\infty}^{2}+\left\|\nabla^{m+1} w_{t}\right\|_{2}^{2}+\left\|\nabla^{\alpha} w_{t}\right\|_{2}^{2}\right) \\
& \leq c E_{m}(v)^{\frac{1}{2}} E_{m}(w) .
\end{aligned}
$$

The term $\left|\left\langle\nabla^{\alpha} M_{5}, \nabla^{\alpha} w_{t}\right\rangle\right|$ is rearranged to

$$
\begin{aligned}
\left\langle\nabla^{\alpha} M_{5}, \nabla^{\alpha} w_{t}\right\rangle & =-\tau\left\langle\left(u \cdot \nabla \nabla^{\alpha}\right) w_{t}, \nabla^{\alpha} w_{t}\right\rangle-\tau\left\langle\nabla^{\alpha}\left((u \cdot \nabla) w_{t}\right)-\left(u \cdot \nabla^{\alpha} \nabla\right) w_{t}, \nabla^{\alpha} w_{t}\right\rangle \\
& \equiv R_{1}+R_{2}
\end{aligned}
$$

and like above $R_{1}=0$ follows.

Moreover one obtains

$$
\begin{aligned}
\left|R_{2}\right| & \leq c \tau\left\|\nabla^{\alpha}\left((u \cdot \nabla) w_{t}\right)-\left(u \cdot \nabla^{\alpha} \nabla\right) w_{t}\right\|_{2}\left\|\nabla^{\alpha} w_{t}\right\|_{2} \\
& \leq c \tau\left(\|\nabla u\|_{\infty}\left\|\nabla^{m} \nabla w_{t}\right\|_{2}+\left\|\nabla w_{t}\right\|_{\infty}\left\|\nabla^{m+1} u\right\|_{2}\right)\left\|\nabla^{\alpha} w_{t}\right\|_{2} \\
& \leq c \tau\left(\|\nabla u\|_{\infty}+\left\|\nabla^{m+1} u\right\|_{2}\right)\left(\left\|\nabla^{m} \nabla w_{t}\right\|_{2}^{2}+\left\|\nabla w_{t}\right\|_{\infty}^{2}+\left\|\nabla^{\alpha} w_{t}\right\|_{2}^{2}\right) \\
& \leq c E_{m}(u)^{\frac{1}{2}} E_{m}(w) .
\end{aligned}
$$

The last term $\left|\left\langle\nabla^{\alpha} M_{6}, \nabla^{\alpha} w_{t}\right\rangle\right|$ can be estimated by

$$
\begin{aligned}
\left|\left\langle\nabla^{\alpha} M_{6}, \nabla^{\alpha} w_{t}\right\rangle\right| & \leq c \tau\left(\|w\|_{\infty}\left\|\nabla^{m+1} \nabla v_{t}\right\|_{2}+\left\|\nabla v_{t}\right\|_{\infty}\left\|\nabla^{m+1} w\right\|_{2}\right)\left\|\nabla^{\alpha} w_{t}\right\|_{2} \\
& \leq c \tau^{2}\left(\left\|\nabla^{m+1} \nabla v_{t}\right\|_{2}^{2}+\left\|\nabla v_{t}\right\|_{m, 2}^{2}\right)\left(\|w\|_{m, 2}^{2}+\left\|\nabla^{m+1} w\right\|_{2}^{2}\right)+\frac{1}{6}\left\|\nabla^{\alpha} w_{t}\right\|_{2}^{2} \\
& \leq c \tau E_{m+1}(v) E_{m}(w)+\frac{1}{6}\left\|\nabla^{\alpha} w_{t}\right\|_{2}^{2} .
\end{aligned}
$$


Now one applies for all $|\alpha| \leq m+1$ the operator $\nabla^{\alpha}$ to (52), but this time multiplies with $\nabla^{\alpha} w$ in $L^{2}\left(\mathbb{R}^{n}\right)$ to the end

$$
\begin{aligned}
\tau \frac{\mathrm{d}}{\mathrm{d} t}\left\langle\nabla^{\alpha} w_{t}, \nabla^{\alpha} w\right\rangle & -\tau\left\|\nabla^{\alpha} w_{t}\right\|_{2}^{2}+\mu\left\|\nabla^{\alpha} \nabla w\right\|_{2}^{2}+\frac{\mathrm{d}}{\mathrm{d} t} \frac{1}{2}\left\|\nabla^{\alpha} w\right\|_{2}^{2} \\
& =-\left\langle\mu \tau \nabla^{\alpha} \Delta v_{t}, \nabla^{\alpha} w\right\rangle+\sum_{j=1}^{6}\left\langle\nabla^{\alpha} M_{j}, \nabla^{\alpha} w\right\rangle .
\end{aligned}
$$

With the same estimates as above one obtains

$$
\begin{aligned}
& \left|\left\langle\nabla^{\alpha} M_{1}, \nabla^{\alpha} w\right\rangle\right| \leq c E_{m}(u)^{\frac{1}{2}} E_{m}(w), \\
& \left|\left\langle\nabla^{\alpha} M_{2}, \nabla^{\alpha} w\right\rangle\right| \leq c E_{m}(v)^{\frac{1}{2}} E_{m}(w), \\
& \left|\left\langle\nabla^{\alpha} M_{3}, \nabla^{\alpha} w\right\rangle\right| \leq c \sqrt{\tau} E_{m}(u)^{\frac{1}{2}} E_{m}(w), \\
& \left|\left\langle\nabla^{\alpha} M_{4}, \nabla^{\alpha} w\right\rangle\right| \leq c E_{m}(v)^{\frac{1}{2}} E_{m}(w) .
\end{aligned}
$$

For the term $\left|\left\langle\nabla^{\alpha} M_{5}, \nabla^{\alpha} w\right\rangle\right|$ one distinguishes between $\alpha=0$ and $\alpha \neq 0$.

For $|\alpha|=0$ it holds

(88) $\left|\left\langle M_{5}, w\right\rangle\right| \leq c \tau\left\|(u \cdot \nabla) w_{t}\right\|_{2}\|w\|_{2} \leq c \tau\|u\|_{\infty}\left\|\nabla w_{t}\right\|_{2}\|w\|_{2} \leq c E_{m}(u)^{\frac{1}{2}} E_{m}(w)$,

while for $|\alpha| \neq 0$ one obtains as above

$$
\begin{aligned}
\left|\left\langle\nabla^{\alpha} M_{5}, \nabla^{\alpha} w\right\rangle\right| & \leq c \tau\left(\|u\|_{\infty}\left\|\nabla^{m} \nabla w_{t}\right\|_{2}+\left\|\nabla w_{t}\right\|_{\infty}\left\|\nabla^{m} u\right\|_{2}\right)\left\|\nabla^{\alpha+e_{k}} w\right\|_{2} \\
& \leq c E_{m}(u)^{\frac{1}{2}} E_{m}(w) .
\end{aligned}
$$

The last term can be estimated to

$$
\begin{aligned}
\left|\left\langle\nabla^{\alpha} M_{6}, \nabla^{\alpha} w\right\rangle\right| & \leq c \tau\left(\|w\|_{\infty}\left\|\nabla^{m+1} \nabla v_{t}\right\|_{2}+\left\|\nabla v_{t}\right\|_{\infty}\left\|\nabla^{m+1} w\right\|_{2}\right)\left\|\nabla^{\alpha} w\right\|_{2} \\
& \leq c \sqrt{\tau} E_{m+1}(v)^{\frac{1}{2}} E_{m}(w) .
\end{aligned}
$$

Now one adds (76) and (83), sums up over $|\alpha| \leq m+1$ and uses the estimates for the nonlinearities (77), (78), (79), (80), (81), (82) and (84), (85), (86), (87), (88) resp. (89), (90) and ends up with

$$
\begin{aligned}
\frac{\mathrm{d}}{\mathrm{d} t} \widetilde{E}_{m}(w)+ & \sum_{|\alpha| \leq m+1}\left(\left\|\nabla^{\alpha} w_{t}\right\|_{2}^{2}-\tau\left\|\nabla^{\alpha} w_{t}\right\|_{2}^{2}+\mu\left\|\nabla^{\alpha} \nabla w\right\|_{2}^{2}\right) \\
\leq & c\left(E_{m}(u)+E_{m+1}(v)+E_{m}(u)^{\frac{1}{2}}+E_{m+1}(v)^{\frac{1}{2}}\right) E_{m}(w) \\
& +\sum_{|\alpha| \leq m+1}\left(\frac{4}{6}\left\|\nabla^{\alpha} w_{t}\right\|_{2}^{2}+\left|\left\langle\mu \tau \nabla^{\alpha} \Delta v_{t}, \nabla^{\alpha} w_{t}\right\rangle\right|+\left|\left\langle\mu \tau \nabla^{\alpha} \Delta v_{t}, \nabla^{\alpha} w\right\rangle\right|\right) .
\end{aligned}
$$

The scalarproducts are estimated by

$$
\begin{aligned}
\mid\left\langle\mu \tau \nabla^{\alpha} \Delta v_{t},\right. & \left.\nabla^{\alpha} w_{t}\right\rangle|+|\left\langle\mu \tau \nabla^{\alpha} \Delta v_{t}, \nabla^{\alpha} w\right\rangle \mid \\
& \leq \mu\left(\frac{3 \mu}{2} \tau^{2}\left\|\nabla^{\alpha} \Delta v_{t}\right\|_{2}^{2}+\frac{1}{6 \mu}\left\|\nabla^{\alpha} w_{t}\right\|_{2}^{2}\right)+\mu\left(\frac{\tau^{2}}{2}\left\|\nabla^{\alpha} \Delta v_{t}\right\|_{2}^{2}+\frac{1}{2}\left\|\nabla^{\alpha} w\right\|_{2}^{2}\right) \\
& \leq c \tau^{2}\left\|\nabla^{\alpha} \Delta v_{t}\right\|_{2}^{2}+\frac{1}{6}\left\|\nabla^{\alpha} w_{t}\right\|_{2}^{2}+c E_{m}(w) .
\end{aligned}
$$


Plugging this in and settig without restriction $\tau \leq \frac{1}{6}$ one finally obtains

$$
\begin{aligned}
\frac{\mathrm{d}}{\mathrm{d} t} \widetilde{E}_{m}(w) \leq & c\left(E_{m}(u)+E_{m+1}(v)+E_{m}(u)^{\frac{1}{2}}+E_{m+1}(v)^{\frac{1}{2}}+1\right) E_{m}(w) \\
& +c \tau^{2} \sum_{|\alpha| \leq m+1}\left\|\nabla^{\alpha} \Delta v_{t}\right\|_{2}^{2}
\end{aligned}
$$

Now one defines $R:=\sum_{|\alpha| \leq m+1}\left\|\nabla^{\alpha} \Delta v_{t}\right\|_{2}^{2}$ and uses (57) what yields (75).

\section{Appendix.}

TheOrem 4.1 (Moser-Inequalities). Let $m \in \mathbb{N}$. Then there is a constant $c=$ $c(m, n)>0$ such that for all $f, g \in W^{m, 2}\left(\mathbb{R}^{n}\right) \cap L^{\infty}\left(\mathbb{R}^{n}\right)$ and $\alpha \in \mathbb{N}_{0}^{n},|\alpha| \leq m$, the following inequalities hold:

$$
\begin{gathered}
\left\|\nabla^{\alpha}(f g)\right\|_{2} \leq c\left(\|f\|_{\infty}\left\|\nabla^{m} g\right\|_{2}+\left\|\nabla^{m} f\right\|_{2}\|g\|_{\infty}\right), \\
\left\|\nabla^{\alpha}(f g)-f \nabla^{\alpha} g\right\|_{2} \leq c\left(\|\nabla f\|_{\infty}\left\|\nabla^{m-1} g\right\|_{2}+\left\|\nabla^{m} f\right\|_{2}\|g\|_{\infty}\right)
\end{gathered}
$$

Proof. see [10, Lemma 4.9].

Lemma 4.2. Let $\alpha, \beta, \gamma \geq 0$. Then

$$
\sup _{t \geq 0} \int_{0}^{t}(1+t-r)^{-\alpha}(1+r)^{-\beta}(1+t)^{\gamma} \mathrm{d} r<\infty
$$

if and only if

(i) $\alpha+\beta-\gamma \geq 1$,

(ii) $\alpha \geq \gamma$ and $\beta \geq \gamma$,

(iii) (if $\beta=1$ then $\alpha>\gamma$ ) and (if $\alpha=1$ then $\beta>\gamma$ ),

are satisfied.

Proof. see [10, Lemma 7.4].

Lemma 4.3 (Nonlinear version of the Lemma from Gronwall). Let $v, w, g \in$ $C^{0}([0, T], \mathbb{R})$

(i) Let $k \in C^{0}([0, T] \times[0, T] \times \mathbb{R}, \mathbb{R}), k(t, s, \cdot)$ be monotone increasing for all $s, t \in$ $[0, T]$ and let

$$
\begin{aligned}
& v(t) \leq g(t)+\int_{0}^{t} k(t, s, v(s)) \mathrm{d} s \\
& w(t) \geq g(t)+\int_{0}^{t} k(t, s, w(s)) \mathrm{d} s \quad \text { for all } t \in[0, T]
\end{aligned}
$$

hold, then

$$
v(t) \leq w(t) \quad \text { for all } t \in[0, T]
$$

holds.

(ii) Let $g \in C^{1}([0, T], \mathbb{R}), k \in C^{1}([0, T] \times \mathbb{R}, \mathbb{R}), k(t, \cdot)$ be monotone increasing for all $t \in[0, T]$ and let $h$ be the local solution of

$$
\begin{aligned}
\dot{h}(t) & =\dot{g}(t)+k(t, h(t)), \\
h(0) & =g(0) .
\end{aligned}
$$


Then for all $v \in C^{0}([0, T], \mathbb{R})$ with

$$
v(t) \leq g(t)+\int_{0}^{t} k(s, v(s)) \mathrm{d} s,
$$

the estimate

$$
v(t) \leq h(t)
$$

holds within the existence time of $h$.

Proof. (i) see [16, Theorem I.1.II].

(ii) cp. $\quad[16$, I.5.IX]. It is well known that $h$ is well defined and $h(t)=g(t)+$ $\int_{0}^{t} k(s, h(s)) \mathrm{d} s$ holds. Therefore part (i) with $w:=h$ applies.

The standard Lemma from Gronwall is included in this lemma (cp. [16, I.1.III]).

\section{REFERENCES}

[1] Y. Brenier, R. NAtAlini, And M. Puel, On a relaxation approximation of the incompressible Navier-Stokes equations, Proceedings of the American Mathematical Society, 132:4 (2003), pp. 1021-1028.

[2] B. Carbonaro And F. Rosso, Some remarks on a modified fluid dynamics equation, Rendiconti Del Circolo Matematico Di Palermo, 2(XXX) (1981), pp. 112-122.

[3] M. Carrassi And A. Morro, A modified Navier-Stokes equation and its consequences on sound dispersion, II Nuovo Cimento 9B(2) (1972).

[4] C. Cattaneo, Sur une forme de l'equation de la chaleur eliminant le paradoxe d'une propagation instantanée, C. R. Acad. Sci. Paris, 247 (1958), pp. 431-433.

[5] M. Dreher, R. Quintanilla, And R. Racke, Ill-posed problems in thermomechanics, Appl. Math. Letters, 22 (2009), pp. 1374-1379.

[6] H. D. Fernández Sare And R. Racke, On the stability of damped Timoshenko systems: Cattaneo versus Fourier law, Arch. Rational Mech. Anal., 194 (2009), pp. 221-251.

[7] G. P. Galdi, An Introduction to the Mathematical Theory of the Navier-Stokes Equations I, 2nd ed., Springer Verlag, 1998.

[8] A. Matsumura, On the Asymptotic Behavior of Solutions of Semi-linear Wave Equations, Publ. RIMS, 12 (1976), pp. 169-189.

[9] M. PAiCu AND G. RAugel, Une perturbation hyperbolique des équations de Navier-Stokes, ESAIM: Proceedings, 21 (2007), pp. 65-87.

[10] R. RACKE, Lectures on nonlinear evolution equations, Intitial value problems, Aspects of Mathematics E19, Friedr. Vieweg \& Sohn, Braunschweig/Wiesbaden (1992).

[11] R. RaCkE AND J. SAAL, Hyperbolic Navier-Stokes equations I: Local well-posedness, Evolution Equations and Control Theory (accepted).

[12] R. RACKE And J. SAAL, Hyperbolic Navier-Stokes equations II: Global existence of small solutions, Evolution Equations and Control Theory (accepted).

[13] A. SchöwE, Langzeitasymptotik der hyperbolischen Navier-Stokes Gleichung in $\mathbb{R}^{3}$, Diploma thesis, University of Konstanz (2011).

[14] H. SoHR, The Navier-Stokes equations. An elementary functional analytic approach, Birkhäuser, 2001.

[15] H. Beirão da Veiga, F. Crispo, and C. R. Grisanti, Reducing slip boundary value problems from the half to the whole space, Applications to inviscid limits and to non-Newtonian fluids, J. Math. Anal. Appl., 377 (2011), pp. 216-227.

[16] W. Walter, Differential and Integral Inequalities, Springer Verlag, 1970. 